\title{
Belgeo
}

Revue belge de géographie

$1 \mid 2017$

Miscellaneous

\section{Jean-Louis Migeot \& Jean-Pierre Coyette, « Silence, on vole! Comprendre la problématique des nuisances sonores aéroportuaires en Belgique »}

\section{Frédéric Dobruszkes}

\section{(2) OpenEdition}

\section{Journals}

Édition électronique

URL : http://journals.openedition.org/belgeo/20773

DOI : 10.4000/belgeo.20773

ISSN : 2294-9135

Éditeur :

National Committee of Geography of Belgium, Société Royale Belge de Géographie

\section{Référence électronique}

Frédéric Dobruszkes, « Jean-Louis Migeot \& Jean-Pierre Coyette, « Silence, on vole! Comprendre la problématique des nuisances sonores aéroportuaires en Belgique » », Belgeo [En ligne], 1 | 2017, mis en ligne le 28 mars 2018, consulté le 24 septembre 2020. URL : http://journals.openedition.org/ belgeo/20773 ; DOI : https://doi.org/10.4000/belgeo.20773

Ce document a été généré automatiquement le 24 septembre 2020.

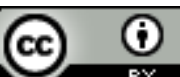

Belgeo est mis à disposition selon les termes de la licence Creative Commons Attribution 4.0 International. 


\title{
Jean-Louis Migeot \& Jean-Pierre Coyette, "Silence, on vole ! Comprendre la problématique des nuisances sonores aéroportuaires en Belgique »
}

\author{
Frédéric Dobruszkes
}

\section{RÉFÉRENCE}

Jean-Louis Migeot \& Jean-Pierre Coyette, « Silence, on vole! Comprendre la problématique des nuisances sonores aéroportuaires en Belgique ", Académie royale de Belgique, octobre 2017, 152 pages ISBN (papier) 978-2-8031-0616-5, ISBN (numérique) 978-2-8031-0617-2

1 On est d'emblée séduit et intrigué par un titre principal - «Silence on vole » - qui sonne fort bien et un sous-titre qui rappelle combien la problématique abordée est d'actualité. Cet ouvrage est écrit par deux docteurs en Sciences appliquées qui enseignent l'acoustique (le premier à l'Université libre de Bruxelles et au Conservatoire royal de Liège, le second à l'Université catholique de Louvain) et ont co-fondé la société Free Field Technologies, qui se présente comme l'un des leaders mondiaux en modélisation des phénomènes sonores et compte notamment Airbus parmi ses clients. C'est dire si ce livre n'est pas un brûlot publié par quelque idéologue et c'est tant mieux, tant le sujet mérite rigueur et analyse dépassionnée.

2 Les auteurs nous proposent une vision plutôt complète du problème. Ils partent de ce qu'est le son (chapitre $1^{\text {er}}$ ) et d'une plongée dans notre appareil auditif (chapitre 2) pour ensuite aborder la délicate question de la mesure du bruit (chapitre 3). Sont ensuite rapidement discutés les sources de bruit (chapitre 4), la certification «bruit » des avions (chapitre 5), les impacts du bruit sur la population (chapitre 6), les moyens 
d'action (chapitre 7) et la réglementation européenne (chapitre 8). Viennent alors les chapitres qui intéresseront probablement le plus les géographes, à savoir l'analyse de la gestion du problème des nuisances sonores des avions induites par les aéroports de Liège et Charleroi (chapitre 9) et de Bruxelles (chapitre 10). Une synthèse (chapitre 11) conclut efficacement l'ouvrage.

3 La comparaison des politiques publiques menées au niveau des deux aéroports wallons, d'une part, et de l'aéroport de Bruxelles, d'autre part, en ressort tout simplement édifiante. À ces trois aéroports, les pouvoirs publics ont encouragé le développement $\mathrm{du}$ trafic aérien. Mais la gestion des nuisances subies par les riverains diverge fortement. À l'aune de la « règle des cinq $\mathrm{C}$ » (Concentration du trafic, Compensation des riverains impactés, Communication, Contrôle et sanction, Continuité), les auteurs concluent que les autorités wallonnes ont, dans une certaine mesure dont on peut certes débattre, pris leurs responsabilités et adopté un ensemble de mesures visant à atténuer l'exposition de la population au bruit des avions. Ceci inclut notamment des plans d'expropriation et d'isolation et un contrôle strict des constructions dans les zones les plus exposées au bruit. C'est tout l'inverse qui est constaté avec l'aéroport de Bruxelles, où les habitants des quartiers densément peuplés sont survolés sans impératif technique et ne sont défendus, in fine, principalement sur la base de l'arrêté «bruit des avions" adopté par le Gouvernement régional bruxellois en 1999 et pleinement appliqué depuis 2017 seulement.

4 Le propos se veut aussi didactique que possible et même le chapitre 3, présenté comme potentiellement "aride» par les auteurs, sera compréhensible pour de nombreux lecteurs. On notera en particulier la riche et intéressante présentation des différentes mesures potentielles du bruit, y compris dans sa dimension temporelle et en insistant sur la différence entre nombre d'événements sonores et indicateurs moyennés. Par contre, les auteurs ne font sans doute pas suffisamment état de la distinction entre la gêne perçue et les impacts sanitaires. L'ouvrage demeure cependant à un niveau suffisamment général et généraliste pour ne pas rebuter les non experts; en fait, il constitue une bonne introduction pour qui n'est pas familier avec le sujet, que ce soit sur le plan de l'acoustique ou sur celui des politiques publiques.

Le revers de la médaille est que le lecteur qui maîtrise déjà le sujet trouvera probablement certains chapitres trop superficiels et pas assez (géo)politiques. C'est en particulier le cas concernant l'adoption par les pouvoirs publics du corpus légal (tant européen que régional) fixant la méthodologie d'évaluation du bruit autour des grands aéroports européens. Comme souvent, les indicateurs sont implicitement présentés comme allant de soi, alors même qu'ils résultent de rapports de force entre intérêts divergents qui s'expriment concrètement par des débats parfois intenses, tant au sein des pouvoirs publics (par exemple entre les pouvoirs exécutif et législatif) et entre les pouvoirs publics et la société (s'exprimant par les lobbies défendant les intérêts du secteur, des associations pro environnement, etc.). Or, de ce point de vue, il est intéressant de noter que les indicateurs de bruit européens (bruit moyen Lden seuillé à $55 \mathrm{~dB}$ et bruit moyen Lnight seuillé à $45 \mathrm{~dB}$ ) ont fait l'objet de vifs débats au sein du Parlement européen et entre le Parlement et le Conseil dans le cadre du processus législatif dit de codécision ${ }^{1}$. Tant la nature de la mesure (bruit moyen vs. événements sonores) que les seuils minima à considérer ont été débattus, de même que l'idée, finalement abandonnée, de fixer des limites de bruit à ne pas dépasser. Symptomatiquement, une grande partie de la contestation récente des nuisances 
aériennes à Bruxelles (elle-même socialement surdéterminée) s'observe à l'extérieur des contours de bruit calculés selon le prescrit européen (Dobruszkes, 2017). Du côté des sciences sociales et politiques, il existe pourtant toute une littérature qui critique l'apparente neutralité des indicateurs prescrits par les pouvoirs publics, en ce compris les indicateurs environnementaux (par exemple Turnhout et al., 2007 ; Rametsteiner et al., 2011). Comme l'écrit Desrosières (1992), "l'information statistique ne tombe pas du ciel comme pur reflet d'une "réalité" antérieure à elle ». Au contraire, et exactement comme les cartes géographiques (Harley, 2001; Crampton et Krygier, 2006), elle construit une réalité et influence ainsi notre vision du monde, au point d'exercer une forme de pouvoir. Mais soyons fair-play : cette approche sociopolitique des indicateurs environnementaux n'a à ce jour guère pénétré le champ des transports.

\section{BIBLIOGRAPHIE}

CRAMPTON J., KRYGIER J. (2006), “An Introduction to Critical Cartography”, ACME: An International E-Journal for Critical Geographies, 4, 1, pp. 11-33.

DESROSIÈRES A. (1992), « Discuter l'indiscutable. Raison statistique et espace public », Raisons pratiques, 3, pp. 131-154.

DOBRUSZKES F. (2017), Assessing noise assessment: EU aircraft noise indicators versus citizen protests, ATARD Workshop, Dublin.

HARLEY J.B. (2001), “Deconstructing the Map”, in LAXTON P., HARLEY J.B., The New Nature of Maps. Essays in the History of Cartography, Baltimore, London, The John Hopkins University Press, pp. 149-168.

RAMETSTEINER E., PÜLZL H., ALKAN-OLSSON J. \& FREDERIKSEN P. (2011), “Sustainability indicator development - Science or political negotiation?", Ecological Indicators 11, 1, pp. 61-70.

TURNHOUT E., HISSCHEMÖLLER M. \& EIJSACKERS H. (2007), "Ecological indicators: Between the two fires of science and policy", Ecological Indicators, 7, 2, pp. 215-228.

\section{NOTES}

1. Depuis lors rebaptisée « procédure législative ordinaire ». 